\title{
$S$-Shaped Connected Component for Nonlinear Fourth-Order Problem of Elastic Beam Equation
}

\author{
Jinxiang Wang, ${ }^{1,2}$ Ruyun Ma, ${ }^{1}$ and Jin Wen ${ }^{1}$ \\ ${ }^{1}$ Department of Mathematics, Northwest Normal University, Lanzhou 730070, China \\ ${ }^{2}$ Department of Applied Mathematics, Lanzhou University of Technology, Lanzhou, China \\ Correspondence should be addressed to Ruyun Ma; mary@nwnu.edu.cn
}

Received 1 December 2016; Accepted 20 June 2017; Published 24 July 2017

Academic Editor: Gennaro Infante

Copyright (c) 2017 Jinxiang Wang et al. This is an open access article distributed under the Creative Commons Attribution License, which permits unrestricted use, distribution, and reproduction in any medium, provided the original work is properly cited.

\begin{abstract}
We investigate the existence of $S$-shaped connected component in the set of positive solutions of the fourth-order boundary value problem: $u^{\prime \prime \prime \prime}(x)=\lambda h(x) f(u(x)), x \in(0,1), u(0)=u(1)=u^{\prime \prime}(0)=u^{\prime \prime}(1)=0$, where $\lambda>0$ is a parameter, $h \in C[0,1]$, and $f \in C[0, \infty)$ with $f_{0}:=\lim _{s \rightarrow 0}(f(s) / s)=\infty$. We develop a bifurcation approach to deal with this extreme situation by constructing a sequence of functions $f^{[n]}$ satisfying $f^{[n]} \rightarrow f$ and $\left(f^{[n]}\right)_{0} \rightarrow \infty$. By studying the auxiliary problems, we get a sequence of unbounded connected components $\mathscr{C}^{[n]}$, and, then, we find an unbounded connected component $\mathscr{C}$ in the set of positive solutions of the fourth-order boundary value problem which satisfies $(0,0) \in \mathscr{C} \subset \lim \sup \mathscr{C}^{[n]}$ and is $S$-shaped.
\end{abstract}

\section{Introduction}

The fourth-order boundary value problem

$$
\begin{aligned}
u^{\prime \prime \prime \prime}(x) & =\lambda f(x, u(x)), \quad x \in(0,1), \\
u(0) & =u(1)=u^{\prime \prime}(0)=u^{\prime \prime}(1)=0
\end{aligned}
$$

describes the deformations of an elastic beam with simple support at the end; see Gupta [1]. This kind of problems has been extensively studied by using topological degree theory, fixed point theorems, lower and upper solutions method, and critical point theory; see [2-17] and the references therein.

Let

$$
f_{0}:=\lim _{s \rightarrow 0} \frac{f(x, s)}{s} .
$$

In this case, $f_{0} \in(0, \infty)$, the global structure of solution set of (1) has been studied extensively by several authors via the well-known Rabinowitz global bifurcation theorem since studying an unbounded connected component bifurcating from the trivial solution at $\left(\pi^{4}, 0\right)$; see Rynne [18], Ma et al.
[19], and Dai and Han [20]. Very recently, Wang and Ma [21] considered the more general problem

$$
\begin{gathered}
u^{\prime \prime \prime \prime}(x)=\lambda f\left(x, u(x), u^{\prime \prime}(x)\right), \quad x \in(0,1), \\
u(0)=u(1)=u^{\prime \prime}(0)=u^{\prime \prime}(1)=0
\end{gathered}
$$

under the following assumption.

(A) There exist constants $a, b \in[0, \infty)$ with $a+b>0$ and $c>0, d>0$ such that

$$
\lim _{\sqrt{s^{2}+p^{2}} \rightarrow 0} \frac{f(x, s, p)-(a s-b p)}{{\sqrt{s^{2}+p^{2}}}^{1+c}}=-d
$$

uniformly for $x \in[0,1]$.

Since $(a+b) \in(0, \infty)$, the Rabinowitz global bifurcation theorem can be used to guarantee an unbounded connected component bifurcating from the trivial solution at $\left(\lambda_{1}(a, b), 0\right)$. However, in the extreme situation $f_{0}=\infty$, the Rabinowitz global bifurcation theorem cannot be directly used to get a connected component bifurcating from the trivial solution anymore. 
To overcome this difficulty, we have to consider a special problem

$$
\begin{gathered}
u^{\prime \prime \prime \prime}(x)=\lambda h(x) f(u(x)), \quad x \in(0,1), \\
u(0)=u(1)=u^{\prime \prime}(0)=u^{\prime \prime}(1)=0
\end{gathered}
$$

where $f_{0}=\infty$. We will develop a bifurcation approach to deal with this extreme situation by constructing a sequence of functions $f^{[n]}$ satisfying

$$
\begin{gathered}
f^{[n]} \longrightarrow f, \\
\left(f^{[n]}\right)_{0} \longrightarrow \infty .
\end{gathered}
$$

By studying the auxiliary problems

$$
\begin{gathered}
u^{\prime \prime \prime \prime}(x)=\lambda h(x) f^{[n]}(u(x)), \quad x \in(0,1), \\
u(0)=u(1)=u^{\prime \prime}(0)=u^{\prime \prime}(1)=0,
\end{gathered}
$$

we get a sequence of unbounded connected components $\mathscr{C}^{[n]}$ of the set of positive solutions of $(1.6)_{n}$, and, then, we find an unbounded component $\mathscr{C}$ in the set of positive solutions of

(5) which satisfies $(0,0) \in \mathscr{C} \subset \lim \sup \mathscr{C}^{[n]}$.

More precisely, we will prove the following.

\section{Theorem 1. Assume that}

(H1) $h \in C[0,1], h(x) \geq 0$ in $[0,1]$, and $h(x)>0$ in $[1 / 4,3 / 4]$;

(H2) $f \in C[0, \infty), f(s)>0$ for all $s>0, f(0)=0$, and $f_{0}:=\lim _{s \rightarrow 0}(f(s) / s)=\infty ;$

(H3) $f_{\infty}:=\lim _{s \rightarrow \infty}(f(s) / s)=0$;

(H4) there exists $s_{0}>0$ such that $0 \leq s \leq s_{0}$ implies that

$$
\begin{aligned}
f(s) & \leq \frac{60}{h^{0}} s_{0}, \\
h^{0} & =\max _{x \in[0,1]} h(x) ;
\end{aligned}
$$

(H5) there exists $s^{0}>s_{0}$ such that

$$
\begin{aligned}
\min _{s \in\left[s^{0}, 4 s^{0}\right]} \frac{f(s)}{s} & \geq \frac{16 \pi^{4}}{h_{0}}, \\
h_{0} & =\min _{x \in[1 / 4,3 / 4]} h(x) .
\end{aligned}
$$

Then there exist $0<\lambda_{*}<1$ and $\lambda^{*}>1$ such that

(i) (5) has at least one positive solution if $0<\lambda<\lambda_{*}$;

(ii) (5) has at least two positive solutions if $\lambda=\lambda_{*}$;

(iii) (5) has at least three positive solutions if $\lambda_{*}<\lambda<\lambda^{*}$;

(iv) (5) has at least two positive solutions if $\lambda=\lambda^{*}$;

(v) (5) has at least one positive solution if $\lambda>\lambda^{*}$.
For other results on the shape of the connected component of solution set, see Wang and Ma [21] and Sim and Tanaka [22].

The rest of this paper is arranged as follows. In Section 2, we give some preliminaries. In Section 3, we consider the corresponding auxiliary problems and obtain a sequence of unbounded components with rightward direction near the initial point. Section 4 is devoted to showing the direction turns of the component, and we complete the proof of Theorem 1.

\section{Preliminaries}

In this section, we state some preliminary results.

Definition 2 (see [23]). Let $X$ be a Banach space and $\left\{\mathscr{C}_{n} \mid\right.$ $n=1,2, \ldots\}$ be a family of subsets of $X$. Then the superior limit $\mathscr{D}$ of $\left\{\mathscr{C}_{n}\right\}$ is defined by

$$
\begin{aligned}
\mathscr{D} & :=\limsup _{n \rightarrow \infty} \mathscr{C}_{n}=\left\{x \in X \mid \exists\left\{n_{i}\right\} \subset \mathbb{N}, x_{n_{i}}\right. \\
& \left.\in \mathscr{C}_{n_{i}} \text {, such that } x_{n_{i}} \longrightarrow x\right\} .
\end{aligned}
$$

Definition 3 (see [23]). A component of a set $Q$ means a maximal connected subset of $Q$.

Lemma 4 (see [24]). Let $X$ be a Banach space, and let $\left\{\mathscr{C}_{n}\right\}$ be a family of connected subsets of $X$. Assume that

(a) there exist $z_{n} \in \mathscr{C}_{n}, n=1,2, \ldots$, and $z^{*} \in X$, such that $z_{n} \rightarrow z^{*}$

(b) $\lim _{n \rightarrow \infty} r_{n}=\infty$, where $r_{n}=\sup \left\{\|x\| \mid x \in \mathscr{C}_{n}\right\}$;

(c) for every $R>0,\left(\bigcup_{n=1}^{\infty} \mathscr{C}_{n}\right) \cap B_{R}$ is a relatively compact set of $X$, where $B_{R}=\{x \in X \mid\|x\| \leq R\}$.

Then there exists an unbounded component $\mathscr{C}$ in $\mathscr{D}$ and $z^{*} \in \mathscr{C}$.

Lemma 5 (see [19, Theorem 2.1]). Assume that (H1) holds. Then the linear problem

$$
\begin{aligned}
u^{\prime \prime \prime \prime}(x) & =\lambda h(x) u(x), \quad x \in(0,1), \\
u(0) & =u(1)=u^{\prime \prime}(0)=u^{\prime \prime}(1)=0
\end{aligned}
$$

has a positive simple principal eigenvalue

$$
\begin{gathered}
\mu_{1}=\inf \left\{\frac{\int_{0}^{1}\left(u^{\prime \prime}(x)\right)^{2} d x}{\int_{0}^{1} h(x) u^{2}(x) d x} \mid u \in C^{4}[0,1], u\right. \\
\left.\quad \not \equiv 0, u(0)=u(1)=u^{\prime \prime}(0)=u^{\prime \prime}(1)=0\right\} .
\end{gathered}
$$

Moreover, the corresponding eigenfunction $\phi$ is positive in $(0,1)$.

Let $g \in C[0,1]$; it is well known that the fourth-order linear problem

$$
\begin{aligned}
& v^{\prime \prime \prime \prime}=g(t), \quad t \in(0,1), \\
& v(0)=v(1)=v^{\prime \prime}(0)=v^{\prime \prime}(1)=0
\end{aligned}
$$


has a unique solution

$$
v(t)=\int_{0}^{1} \int_{0}^{1} G(t, s) G(s, \tau) g(\tau) d \tau d s,
$$

where

$$
G(t, s)= \begin{cases}s(1-t), & 0 \leq s \leq t \leq 1 \\ t(1-s), & 0 \leq t \leq s \leq 1\end{cases}
$$

Moreover, if $g \geq 0$ and $g \neq 0$, then

$$
v^{\prime \prime}(t)=-\int_{0}^{1} G(t, s) g(s) d s \leq 0
$$

that is, $v \geq 0$ is concave.

Since Green's function $G(t, s)$ has the properties (i) $0 \leq G(t, t) G(s, s) \leq G(t, s) \leq G(s, s), \forall t, s \in(0,1)$,

(ii) $G(t, s) \geq(1 / 4) G(s, s), \forall t \in[1 / 4,3 / 4], s \in(0,1)$,

then, for any $t \in[1 / 4,3 / 4]$, we have

$$
\begin{aligned}
v(t) & =\int_{0}^{1} G(t, s)\left[\int_{0}^{1} G(s, \tau) g(\tau) d \tau\right] d s \\
& \geq \frac{1}{4} \int_{0}^{1} G(s, s)\left[\int_{0}^{1} G(s, \tau) g(\tau) d \tau\right] d s \\
& \geq \frac{1}{4}\|v\|_{\infty} .
\end{aligned}
$$

\section{Auxiliary Problems and Rightward Bifurcation}

For each $n \in \mathbb{N}$, we define $f^{[n]}(s):[0, \infty) \rightarrow[0, \infty)$ by

$$
f^{[n]}(s)= \begin{cases}f(s), & \text { if } s \in\left(\frac{1}{n}, \infty\right) \\ s\left[2 n f\left(\frac{1}{n}\right)+(2 n)^{-\alpha}-2 n f\left(\frac{1}{2 n}\right)\right]+\left[2 f\left(\frac{1}{2 n}\right)-2(2 n)^{-1-\alpha}-f\left(\frac{1}{n}\right)\right], & \text { if } s \in\left(\frac{1}{2 n}, \frac{1}{n}\right], \\ 2 n f\left(\frac{1}{2 n}\right) s-s^{1+\alpha}, & \text { if } s \in\left[0, \frac{1}{2 n}\right] .\end{cases}
$$

Then $f^{[n]} \in C([0, \infty),[0, \infty))$ with $f^{[n]}(s)>0$ for all $s \in$ $(0, \infty)$ and

$$
\left(f^{[n]}\right)_{0}:=\lim _{s \rightarrow 0} \frac{f^{[n]}(s)}{s}=2 n f\left(\frac{1}{2 n}\right)>0 .
$$

By (H2), it follows that $\lim _{n \rightarrow \infty}\left(f^{[n]}\right)_{0}=\infty$.

We extend $f$ to an odd function $g: \mathbb{R} \rightarrow \mathbb{R}$ by

$$
g(s)= \begin{cases}f(s), & \text { if } s \geq 0 \\ -f(-s), & \text { if } s<0\end{cases}
$$

Similarly we may extend $f^{[n]}$ to an odd function $g^{[n]}: \mathbb{R} \rightarrow \mathbb{R}$ for each $n \in \mathbb{N}$.

Now let us consider the auxiliary family of the equations

$$
\begin{gathered}
u^{\prime \prime \prime \prime}(x)=\lambda h(x) g^{[n]}(u(x)), \quad x \in(0,1), \\
u(0)=u(1)=u^{\prime \prime}(0)=u^{\prime \prime}(1)=0 .
\end{gathered}
$$

We rewrite $\left(P_{n}\right)$ by

$$
\begin{aligned}
u^{\prime \prime \prime \prime}(x)= & \lambda h(x)\left(g^{[n]}\right)_{0} u(x) \\
& +\lambda h(x)\left[g^{[n]}(u(x))-\left(g^{[n]}\right)_{0} u(x)\right], \\
x \in(0,1), & \\
u(0)= & u(1)=u^{\prime \prime}(0)=u^{\prime \prime}(1)=0 .
\end{aligned}
$$

Since (18) implies $\left(g^{[n]}\right)_{0}=2 n f(1 / 2 n)>0$, then, using Rabinowitz's global bifurcation theorem and following the similar arguments in the proof of Theorem 1.1 in [19] or Theorem 2.2 in [20], we have the following.

Lemma 6. Assume that (H1) and (H2) hold; then, for each fixed $n \in \mathbb{N}$, from $\left(\mu_{1} /\left(g^{[n]}\right)_{0}, 0\right)$ there emanates an unbounded subcontinuum $\mathscr{C}^{[n]}$ of positive solutions of $\left(P_{n}\right)$ in the set $\mathbb{R} \times E$, where $E=\left\{u \in C^{3}[0,1] \mid u(0)=u(1)=u^{\prime \prime}(0)=u^{\prime \prime}(1)=0\right\}$ with the norm $\|u\|=\|u\|_{\infty}+\left\|u^{\prime}\right\|_{\infty}+\left\|u^{\prime \prime}\right\|_{\infty}+\left\|u^{\prime \prime \prime}\right\|_{\infty}$.

Lemma 7. Assume that (H1) and (H2) hold. For each fixed $n \in \mathbb{N}$, let $\left\{\left(\lambda_{k}, u_{k}\right) \mid k=1,2, \ldots\right\}$ be a sequence of positive solutions to $\left(P_{n}\right)$ which satisfies $\lambda_{k} \rightarrow \mu_{1} /\left(g^{[n]}\right)_{0}$ and $\left\|u_{k}\right\| \rightarrow$ 0 as $k \rightarrow \infty$. Let $\phi$ be the first eigenfunction of (10) which satisfies $\|\phi\|_{\infty}=1$. Then there exists a subsequence of $\left\{u_{k}\right\}$, again denoted by $\left\{u_{k}\right\}$, such that $u_{k} /\left\|u_{k}\right\|_{\infty}$ converges uniformly to $\phi$ on $[0,1]$.

Proof. Set $v_{k}:=u_{k} /\left\|u_{k}\right\|_{\infty}$. Then $\left\|v_{k}\right\|_{\infty}=1$. For every $\left(\lambda_{k}, u_{k}\right)$, we have

$$
u_{k}^{\prime \prime}(x)=-\lambda_{k} \int_{0}^{1} G(x, s) h(s) g^{[n]}\left(u_{k}(s)\right) d s
$$

From the boundary condition $u_{k}(0)=u_{k}(1)=0$, there exists $\widehat{x}_{k} \in(0,1)$ such that $u_{k}^{\prime}\left(\widehat{x}_{k}\right)=0$. Integrating $(21)$ on $\left[x, \widehat{x}_{k}\right]$, we obtain 


$$
u_{k}^{\prime}(x)=\lambda_{k} \int_{x}^{\widehat{x}_{k}} \int_{0}^{1} G(t, s) h(s) g^{[n]}\left(u_{k}(s)\right) d s d t
$$
$x \in[0,1]$.

Dividing both sides of (22) by $\left\|u_{k}\right\|_{\infty}$, we get

$$
\begin{aligned}
& v_{k}^{\prime}(x) \\
& =\lambda_{k} \int_{x}^{\widehat{x}_{k}} \int_{0}^{1} G(t, s) h(s) \frac{g^{[n]}\left(u_{k}(s)\right)}{u_{k}(s)} v_{k}(s) d s d t, \\
& x \in[0,1] .
\end{aligned}
$$

Since $\left\|u_{k}\right\| \rightarrow 0$ implies $\left\|u_{k}\right\|_{\infty} \rightarrow 0$, then, by (18), there exists a constant $m_{1}>2 n f(1 / 2 n)$ such that

$$
\frac{g^{[n]}\left(u_{k}(s)\right)}{u_{k}(s)}<m_{1}, \quad \forall k \in \mathbb{N}, s \in(0,1) .
$$

From $\lambda_{k} \rightarrow \mu_{1} /\left(g^{[n]}\right)_{0}$, it follows that there exists a constant $m_{2}>0$ such that

$$
\lambda_{k} \leq m_{2}, \quad \forall k \in \mathbb{N}
$$

Then, for $x \in[0,1],(23)$ implies that

$$
\begin{aligned}
& \lambda_{k} \int_{x}^{\widehat{x}_{k}} \int_{0}^{1} G(t, s) h(s) \frac{g^{[n]}\left(u_{k}(s)\right)}{u_{k}(s)} v_{k}(s) d s d t \\
& \quad \leq m_{1} m_{2}\left\|v_{k}\right\|_{\infty} \int_{x}^{\widehat{x}_{k}} \int_{0}^{1} G(t, s) h(s) d s d t \\
& \quad \leq m_{1} m_{2}\left\|v_{k}\right\|_{\infty} \int_{0}^{1} \int_{0}^{1} G(t, s) h(s) d s d t \\
& =M\left\|v_{k}\right\|_{\infty}=M, \\
& \quad\left(M=m_{1} m_{2} \int_{0}^{1} \int_{0}^{1} G(t, s) h(s) d s d t\right) ;
\end{aligned}
$$

that is,

$$
\left\|v_{k}^{\prime}\right\|_{\infty} \leq M, \quad \forall k \in \mathbb{N}
$$

Since $\left\|v_{k}^{\prime}\right\|_{\infty}$ is bounded, by the Ascoli-Arzela theorem, a subsequence of $\left\{v_{k}\right\}$ uniformly converges to a limit $v \in C[0,1]$ with $\|v\|_{\infty}=1$, and we again denote by $\left\{v_{k}\right\}$ the subsequence.

For every $\left(\lambda_{k}, u_{k}\right)$, we have

$$
\begin{aligned}
& u_{k}(x)=\lambda_{k} \int_{0}^{1} G(x, s) \\
& \cdot\left[\int_{0}^{1} G(s, \tau) h(\tau) g^{[n]}\left(u_{k}(\tau)\right) d \tau\right] d s .
\end{aligned}
$$

Dividing both sides of (28) by $\left\|u_{k}\right\|_{\infty}$, we get

$$
\begin{aligned}
& v_{k}(x)=\lambda_{k} \int_{0}^{1} G(x, s) \\
& \cdot\left[\int_{0}^{1} G(s, \tau) h(\tau) \frac{g^{[n]}\left(u_{k}(\tau)\right)}{u_{k}(\tau)} v_{k}(\tau) d \tau\right] d s .
\end{aligned}
$$

Since $\left\|u_{k}\right\|_{\infty} \rightarrow 0$, we conclude that $g^{[n]}\left(u_{k}(\tau)\right) / u_{k}(\tau) \rightarrow$ $\left(g^{[n]}\right)_{0}$ for each fixed $\tau \in[0,1]$. Then Lebesgue's dominated convergence theorem shows that

$$
\begin{aligned}
& v(x)=\frac{\mu_{1}}{\left(g^{[n]}\right)_{0}} \\
& \cdot \int_{0}^{1} G(x, s)\left[\int_{0}^{1} G(s, \tau) h(\tau)\left(g^{[n]}\right)_{0} v(\tau) d \tau\right] d s \\
& =\mu_{1} \int_{0}^{1} G(x, s)\left[\int_{0}^{1} G(s, \tau) h(\tau) v(\tau) d \tau\right] d s,
\end{aligned}
$$

which means that $v$ is a nontrivial solution of (10) with $\lambda=\mu_{1}$, and hence $v \equiv \phi$.

Lemma 8. Assume that (H1) and (H2) hold. For each fixed $n \in \mathbb{N}$, let $\mathscr{C}^{[n]}$ be as in Lemma 6 . Then there exists $\delta>0$ such that $(\lambda, u) \in \mathscr{C}^{[n]}$ and $\left|\lambda-\mu_{1} /\left(g^{[n]}\right)_{0}\right|+\|u\| \leq \delta$ imply $\lambda>\mu_{1} /\left(g^{[n]}\right)_{0}$.

Proof. Assume to the contrary that there exists a sequence $\left\{\left(\lambda_{k}, u_{k}\right) \mid k=1,2, \ldots\right\} \subset \mathscr{C}^{[n]}$ such that $\lambda_{k} \rightarrow \mu_{1} /\left(g^{[n]}\right)_{0}$, $\left\|u_{k}\right\| \rightarrow 0$, and $\lambda_{k} \leq \mu_{1} /\left(g^{[n]}\right)_{0}$. By Lemma 7 , there exists a subsequence of $\left\{u_{k}\right\}$, again denoted by $\left\{u_{k}\right\}$, such that $u_{k} /\left\|u_{k}\right\|_{\infty}$ converges uniformly to $\phi$ on $[0,1]$. Multiplying the equation of $\left(P_{n}\right)$ with $(\lambda, u)=\left(\lambda_{k}, u_{k}\right)$ by $\phi$ and integrating it over $[0,1]$, we have

$$
\begin{aligned}
& \int_{0}^{1} \phi(x) u_{k}^{\prime \prime \prime \prime}(x) d x \\
& \quad=\lambda_{k} \int_{0}^{1} h(x) g^{[n]}\left(u_{k}(x)\right) \phi(x) d x
\end{aligned}
$$

By simple computation, one has that

$$
\begin{aligned}
\int_{0}^{1} \phi(x) u_{k}^{\prime \prime \prime \prime}(x) d x & =\int_{0}^{1} \phi^{\prime \prime \prime \prime}(x) u_{k}(x) d x \\
& =\mu_{1} \int_{0}^{1} h(x) \phi(x) u_{k}(x) d x .
\end{aligned}
$$

Combining (31) with (32), we obtain

$$
\begin{aligned}
& \int_{0}^{1} h(x) g^{[n]}\left(u_{k}(x)\right) \phi(x) d x \\
& \quad=\frac{\mu_{1}}{\lambda_{k}} \int_{0}^{1} h(x) \phi(x) u_{k}(x) d x
\end{aligned}
$$

that is

$$
\begin{aligned}
& \frac{\int_{0}^{1} h(x) \phi(x)\left[g^{[n]}\left(u_{k}(x)\right)-\left(g^{[n]}\right)_{0} u_{k}(x)\right] d x}{\left\|u_{k}\right\|_{\infty}^{1+\alpha}} \\
& =\frac{\int_{0}^{1} h(x) \phi(x)\left[\left(\mu_{1} / \lambda_{k}\right) u_{k}(x)-\left(g^{[n]}\right)_{0} u_{k}(x)\right] d x}{\left\|u_{k}\right\|_{\infty}^{1+\alpha}} .
\end{aligned}
$$


Since $\left\|u_{k}\right\| \rightarrow 0$ implies $\left\|u_{k}\right\|_{\infty} \rightarrow 0$, then, from the definition of $g^{[n]}$ and (17), we have

$$
\lim _{k \rightarrow \infty} \frac{g^{[n]}\left(u_{k}(x)\right)-\left(g^{[n]}\right)_{0} u_{k}(x)}{\left(u_{k}(x)\right)^{1+\alpha}}=-1,
$$

$\forall x \in(0,1)$.

Then Lebesgue's dominated convergence theorem, Lemma 7, and (35) imply that

$$
\begin{aligned}
& \frac{\int_{0}^{1} h(x) \phi(x)\left[g^{[n]}\left(u_{k}(x)\right)-\left(g^{[n]}\right)_{0} u_{k}(x)\right] d x}{\left\|u_{k}\right\|_{\infty}^{1+\alpha}} \\
& =\int_{0}^{1} h(x) \phi(x) \\
& \quad \cdot \frac{g^{[n]}\left(u_{k}(x)\right)-\left(g^{[n]}\right)_{0} u_{k}(x)}{\left(u_{k}(x)\right)^{1+\alpha}}\left[\frac{u_{k}(x)}{\left\|u_{k}\right\|_{\infty}}\right]^{1+\alpha} d x \longrightarrow \\
& -\int_{0}^{1} h(x) \phi^{2+\alpha}(x) d x<0,
\end{aligned}
$$

$$
=\frac{\mu_{1}-\left(g^{[n]}\right)_{0} \lambda_{k}}{\lambda_{k}\left\|u_{k}\right\|_{\infty}^{\alpha}} \int_{0}^{1} h(x) \phi(x) \frac{u_{k}(x)}{\left\|u_{k}\right\|_{\infty}} d x ;
$$

by Lebesgue's dominated convergence theorem and Lemma 7 again, we conclude that

$$
\begin{aligned}
\int_{0}^{1} h(x) \phi(x) \frac{u_{k}(x)}{\left\|u_{k}\right\|_{\infty}} d x \rightarrow & \\
& \int_{0}^{1} h(x) \phi^{2}(x) d x>0, \text { as } k \longrightarrow \infty
\end{aligned}
$$

this contradicts (34).

\section{Direction Turns of Component and Proof of Theorem 1}

Lemma 9. Assume that (H1) and (H2) hold; then there exists an unbounded connected component $\mathscr{C} \subset \mathbb{R} \times E$ with $(0,0) \in \mathscr{C}$ in the solutions set of (5). Moreover, the component $\mathscr{C}$ grows to the right near $(0,0)$.

Proof. Let us verify that $\left\{\mathscr{C}^{[n]} \mid n=1,2, \ldots\right\}$ satisfy all of the conditions of Lemma 4.

Since

$$
\lim _{n \rightarrow \infty} \frac{\mu_{1}}{\left(g^{[n]}\right)_{0}}=\lim _{n \rightarrow \infty} \frac{\mu_{1}}{2 n f(1 / 2 n)}=0,
$$

Condition (a) in Lemma 4 is satisfied with $z^{*}=(0,0)$. Obviously

$$
r_{n}=\sup \left\{|\lambda|+\|u\| \mid(\lambda, u) \in \mathscr{C}^{[n]}\right\}=\infty
$$

and, accordingly, (b) holds. (c) can be deduced directly from the Ascoli-Arzela theorem and the definition of $g^{[n]}$. Therefore, the superior limit of $\left\{\mathscr{C}^{[n]}\right\}$, that is, $\mathscr{D}$, contains an unbounded connected component $\mathscr{C} \subset \mathbb{R} \times E$ with $(0,0) \in$ $\mathscr{C}$. From Lemma 8 , the component $\mathscr{C}$ grows to right near $(0,0)$.

Lemma 10. Assume that (H1), (H2), and (H3) hold. Let $\left\{\left(\lambda_{k}, u_{k}\right) \mid k=1,2, \ldots\right\} \subset \mathscr{C} \subset \mathbb{R} \times E$ be a sequence of positive solutions to (5); then $\left\|u_{k}\right\| \rightarrow \infty$ implies $\left\|u_{k}\right\|_{\infty} \rightarrow \infty$.

Proof. Assume on the contrary that $\left\|u_{k}\right\|_{\infty}$ is bounded; we divide the proof into two cases.

Case 1 $\left(\left\{\left(\lambda_{k}\right)\right\}\right.$ Is Bounded). By recalling (22) and (21), we have that $\left\|u_{k}^{\prime}\right\|_{\infty}$ and $\left\|u_{k}^{\prime \prime}\right\|_{\infty}$ are bounded.

From the boundary condition $u_{k}^{\prime \prime}(0)=u_{k}^{\prime \prime}(1)=0$, there exists $x_{k}^{*} \in(0,1)$ such that $u_{k}^{\prime \prime \prime}\left(x_{k}^{*}\right)=0$. Integrating the equation of (5) on $\left[x_{k}^{*}, x\right]$, we obtain

$$
\begin{array}{r}
u_{k}^{\prime \prime \prime}(x)=\int_{x_{k}^{*}}^{x} u_{k}^{\prime \prime \prime \prime}(s) d s=\lambda_{k} \int_{x_{k}^{*}}^{x} h(s) f\left(u_{k}(s)\right) d s, \\
x \in[0,1] ;
\end{array}
$$

then $\left\|u_{k}^{\prime \prime \prime}\right\|_{\infty}$ is bounded too. Finally, we conclude that $\left\|u_{k}\right\|=$ $\left\|u_{k}\right\|_{\infty}+\left\|u_{k}^{\prime}\right\|_{\infty}+\left\|u_{k}^{\prime \prime}\right\|_{\infty}+\left\|u_{k}^{\prime \prime \prime}\right\|_{\infty}$ is bounded; this deduces a contradiction.

Case $2\left(\lambda_{k} \rightarrow \infty\right)$. Since $\left\|u_{k}\right\|_{\infty}$ is bounded, then, by (H2) and (H3), there exists constant $C>0$ such that

$$
f\left(u_{k}(x)\right) \geq C u_{k}(x), \quad \forall k \in \mathbb{N}, x \in(0,1) .
$$

Since $\left(\lambda_{k}, u_{k}\right) \in \mathscr{C}$, combining (42) with (16) we have

$$
\begin{aligned}
u_{k}(x)=\lambda_{k} \int_{0}^{1} G(x, s) \\
.\left[\int_{0}^{1} G(s, \tau) h(\tau) f\left(u_{k}(\tau)\right) d \tau\right] d s \\
\geq \lambda_{k} \int_{0}^{1} G(x, s) \\
\cdot\left[\int_{1 / 4}^{3 / 4} G(s, \tau) h(\tau) C u_{k}(\tau) d \tau\right] d s \geq C \\
\cdot \frac{1}{4}\left\|u_{k}\right\|_{\infty} \lambda_{k} \int_{0}^{1} G(x, s) \\
.\left[\int_{1 / 4}^{3 / 4} G(s, \tau) h(\tau) d \tau\right] d s
\end{aligned}
$$

which yields that $\left\{\lambda_{k}\right\}$ is bounded; this deduces a contradiction. 
Lemma 11. Assume that (H1), (H2), and (H3) hold. Then, $\mathscr{C}$ joins $(0,0)$ to $(\infty, \infty)$ in $[0, \infty) \times E$.

Proof. We divide the proof into two steps.

Step 1. We show that $\sup \{\lambda \mid(\lambda, u) \in \mathscr{C}\}=\infty$.

Assume on the contrary that $\sup \{\lambda \mid(\lambda, u) \in \mathscr{C}\}=: c_{0}<$ $\infty$. Let $\left\{\left(\lambda_{k}, u_{k}\right) \mid k=1,2, \ldots\right\} \subset \mathscr{C}$ be such that $\left|\lambda_{k}\right|+\left\|u_{k}\right\| \rightarrow$ $\infty$; then $\left\|u_{k}\right\| \rightarrow \infty$, and from Lemma $10,\left\|u_{k}\right\|_{\infty} \rightarrow \infty$.

Since $\left(\lambda_{k}, u_{k}\right) \in \mathscr{C}$, we have that

$$
\begin{gathered}
u_{k}^{\prime \prime \prime \prime}(x)=\lambda_{k} h(x) f\left(u_{k}(x)\right), \quad x \in(0,1), \\
u_{k}(0)=u_{k}(1)=u_{k}^{\prime \prime}(0)=u_{k}^{\prime \prime}(1)=0 .
\end{gathered}
$$

Set $\omega_{k}:=u_{k} /\left\|u_{k}\right\|_{\infty}$, and then $\left\|\omega_{k}\right\|_{\infty}=1$, and

$$
\begin{gathered}
\omega_{k}^{\prime \prime \prime \prime}(x)=\frac{\lambda_{k} h(x) f\left(u_{k}(x)\right)}{\left\|u_{k}\right\|_{\infty}}, \quad x \in(0,1), \\
\omega_{k}(0)=\omega_{k}(1)=\omega_{k}^{\prime \prime}(0)=\omega_{k}^{\prime \prime}(1)=0 .
\end{gathered}
$$

From (H3), we have that $\lambda_{k} h(x) f\left(u_{k}(x)\right) /\left\|u_{k}\right\|_{\infty}$ is bounded uniformly; then $\left\{\omega_{k}^{\prime \prime \prime \prime}\right\}$ is bounded. By Ascoli-Arzela theorem, choosing a subsequence and relabelling it if necessary, it follows that there exists $(\widehat{\lambda}, \widehat{u}) \in\left[0, c_{0}\right] \times E$ with $\|\widehat{u}\|_{\infty}=1$ such that

$$
\lim _{k \rightarrow \infty}\left(\lambda_{k}, \omega_{k}\right)=(\widehat{\lambda}, \widehat{u})
$$

Let

$$
\tilde{f}(r)=\max \{f(s)|0 \leq| s \mid \leq r\} ;
$$

then $\tilde{f}$ is nondecreasing and (H3) implies that

$$
\lim _{r \rightarrow \infty} \frac{\tilde{f}(r)}{r}=0 .
$$

Since

$$
\frac{f\left(u_{k}(x)\right)}{\left\|u_{k}\right\|_{\infty}} \leq \frac{\tilde{f}\left(\left\|u_{k}\right\|_{\infty}\right)}{\left\|u_{k}\right\|_{\infty}},
$$

this together with (48) and $\left\|u_{n}\right\|_{\infty} \rightarrow \infty$ implies that

$$
\lim _{k \rightarrow \infty} \frac{f\left(u_{k}(x)\right)}{\left\|u_{k}\right\|_{\infty}}=0, \quad \text { uniformly for } x \in[0,1] .
$$

Notice that (45) is equivalent to

$$
\begin{aligned}
& \omega_{k}(x) \\
& =\lambda_{k} \int_{0}^{1} G(x, s)\left[\int_{0}^{1} G(s, \tau) h(\tau) \frac{f\left(u_{k}(\tau)\right)}{\left\|u_{k}\right\|_{\infty}} d \tau\right] d s, \\
& x \in(0,1) .
\end{aligned}
$$

Combining this with (50) and using (46) and Lebesgue dominated convergence theorem, it follows that

$$
\widehat{u}=\widehat{\lambda} \int_{0}^{1} G(x, s)\left[\int_{0}^{1} G(s, \tau) h(\tau) 0 d \tau\right] d s=0,
$$

This contradicts with $\|\widehat{u}\|_{\infty}=1$. Therefore, $\sup \{\lambda \mid(\lambda, u) \epsilon$ $\mathscr{C}\}=\infty$.

Step 2. We show that $\sup \{\|u\| \mid(\lambda, u) \in \mathscr{C}\}=\infty$.

Assume on the contrary that $\sup \{\|u\| \mid(\lambda, u) \in \mathscr{C}\}=$ : $M_{0}<\infty$. Let $\left\{\left(\lambda_{k}, u_{k}\right)\right\} \subset \mathscr{C}$ be such that

$$
\begin{aligned}
& \lambda_{k} \longrightarrow \infty, \\
&\left\|u_{k}\right\| \leq M_{0} .
\end{aligned}
$$

Since $\left\|u_{k}\right\| \leq M_{0}$ implies $\left\|u_{k}\right\|_{\infty} \leq M_{0}$, then, following the same arguments in the proof of Case 2 in Lemma 10, we can get a contradiction.

Lemma 12. Assume that (H1), (H2), (H3), and (H4) hold. Let $(\lambda, u) \in \mathscr{C}$ be a solution of (5) with $\|u\|_{\infty}=s_{0}$; then $\lambda>1$.

Proof. Let $u$ be a solution of (5) with $\|u\|_{\infty}=s_{0}$; then, by Condition (H4) and the property of $G(x, s)$, we have

$$
\begin{aligned}
s_{0} & =\|u\|_{\infty}=\max _{x \in[0,1]}\left\{\lambda \int_{0}^{1} G(x, s)\right. \\
\cdot & {\left.\left[\int_{0}^{1} G(s, \tau) h(\tau) f(u(\tau)) d \tau\right] d s\right\}<\lambda h^{0} \frac{60}{h^{0}} } \\
\cdot & s_{0} \int_{0}^{1} G(s, s)\left[\int_{0}^{1} G(s, \tau) d \tau\right] d s=\lambda s_{0}
\end{aligned}
$$

then $\lambda>1$.

Lemma 13. Assume that (H1), (H2), (H3), (H4), and (H5) hold. Let $(\lambda, u) \in \mathscr{C}$ be a solution of (5) with $\|u\|_{\infty}=4 s^{0}$; then $\lambda<1$.

Proof. Let $u$ be a solution of (5) with $\|u\|_{\infty}=4 s^{0}$; then (16) implies that

$$
s^{0}=\frac{1}{4}\|u\|_{\infty} \leq u(x) \leq\|u\|_{\infty}=4 s_{0}, \quad x \in\left[\frac{1}{4}, \frac{3}{4}\right] .
$$

Suppose on the contrary that $\lambda \geq 1$; then, by (H5) we have

$$
\begin{aligned}
u^{\prime \prime \prime \prime}(x) & =\lambda h(x) f(u(x)) \geq \lambda h(x) \frac{f(u(x))}{u(x)} u(x) \\
& \geq 16 \pi^{4} u(x), \quad x \in\left[\frac{1}{4}, \frac{3}{4}\right] .
\end{aligned}
$$

Multiplying inequality (56) by $\sin [2 \pi(x-1 / 4)]$ and integrating it over $[1 / 4,3 / 4]$, we have

$$
\begin{aligned}
& \int_{1 / 4}^{3 / 4} u^{\prime \prime \prime \prime} \sin \left[2 \pi\left(x-\frac{1}{4}\right)\right] d x \\
& \quad \geq \int_{1 / 4}^{3 / 4} 16 \pi^{4} u(x) \sin \left[2 \pi\left(x-\frac{1}{4}\right)\right] d x .
\end{aligned}
$$

$$
x \in(0,1) \text {. }
$$


On the other hand, by simple computation, one has that

$$
\begin{aligned}
& \int_{1 / 4}^{3 / 4} u^{\prime \prime \prime \prime} \sin \left[2 \pi\left(x-\frac{1}{4}\right)\right] d x \\
& =2 \pi\left[u^{\prime \prime}\left(\frac{1}{4}\right)+u^{\prime \prime}\left(\frac{3}{4}\right)\right] \\
& -8 \pi^{3}\left[u\left(\frac{1}{4}\right)+u\left(\frac{3}{4}\right)\right] \\
& +\int_{1 / 4}^{3 / 4} 16 \pi^{4} u(x) \sin \left[2 \pi\left(x-\frac{1}{4}\right)\right] d x ;
\end{aligned}
$$

since $u(t) \geq 0$ is concave, then $2 \pi\left[u^{\prime \prime}(1 / 4)+u^{\prime \prime}(3 / 4)\right]-$ $8 \pi^{3}[u(1 / 4)+u(3 / 4)]<0$; this deduces a contradiction.

Proof of Theorem 1. From Lemma 9, there exists an unbounded connected component $\mathscr{C}$ in the positive solutions set of (5); moreover, $\mathscr{C} \subset \mathbb{R} \times E$ with $(0,0) \in \mathscr{C}$ and it grows to the right near $(0,0)$. From Lemma 11 , there exists a sequence $\left\{\left(\lambda_{k}, u_{k}\right)\right\} \subset \mathscr{C}$ such that $\lambda_{k} \rightarrow \infty$ and $\left\|u_{k}\right\| \rightarrow \infty$. Lemma 10 implies that $\left\|u_{k}\right\|_{\infty} \rightarrow \infty$; then there exist $\left(\lambda_{0}, u_{0}\right)$ and $\left(\lambda^{0}, u^{0}\right) \in \mathscr{C}$ such that $\left\|u_{0}\right\|_{\infty}=s_{0}$ and $\left\|u^{0}\right\|_{\infty}=4 s^{0}$; Lemmas 12 and 13 imply that $\lambda_{0}>1$ and $\lambda^{0}<1$, respectively.

By Lemmas 11, 12, and 13, there exist $\left(\lambda^{*}, u^{*}\right)$ and $\left(\lambda_{*}, u_{*}\right) \in \mathscr{C}$ which satisfy $0<\lambda_{*}<1<\lambda^{*}$ and $\left\|u^{*}\right\|<\left\|u_{*}\right\|$, such that the component $\mathscr{C}$ turns to the left at $\left(\lambda^{*}, u^{*}\right)$ and to the right at $\left(\lambda_{*}, u_{*}\right)$; that is, $\mathscr{C}$ is an $S$-shaped component; this together with Lemma 9 completes the proof of Theorem 1 .

Remark 14. Let us take

$$
f(x, s, p) \equiv h(x) f(s) ;
$$

then Condition (A) in [21] (see (4)) implies

$$
f_{0}=a \in(0, \infty)
$$

which means $f$ is in linear growth at zero. Equation (60) guarantees that Rabinowitz global bifurcation theorem can be directly used to bifurcate a connected component from $\left(\lambda_{1} / f_{0}, 0\right)$. However, in this paper, we deal with (5) under the superlinear growth condition at zero; that is,

$$
\text { (H2) } f_{0}:=\lim _{s \rightarrow 0}(f(s) / s)=\infty \text {. }
$$

In the situation, $f_{0}=\infty$, the Rabinowitz global bifurcation theorem cannot be directly used to get a connected component joining $(0,0)$ with infinity anymore. To overcome this difficulty, we have to construct a sequence of functions $f^{[n]}$ which is in linear growth at zero and satisfies

$$
\begin{aligned}
\lim _{n \rightarrow \infty} f^{[n]} & \longrightarrow f, \\
\lim _{n \rightarrow \infty}\left(f^{[n]}\right)_{0} & =\infty .
\end{aligned}
$$

By studying the corresponding auxiliary problems $(1.6)_{n}$, we obtain a sequence of unbounded connected components $\left\{\mathscr{C}^{[n]}\right\}$ via Rabinowitz global bifurcation theorem. Now, by using the fact that the superior limit of certain infinity collection of connected components contains an unbounded connected component (see Ma and An in [24]), we get a connected component $\mathscr{C}$ :

$$
(0,0) \in \mathscr{C} \subset \limsup _{n \rightarrow \infty} \mathscr{C}^{[n]}
$$

which joins $(0,0)$ with infinity.

Therefore, the key conditions, the conclusion, and the proofs of the main results in this paper and in [21] are very different.

\section{Conflicts of Interest}

The authors declare that there are no conflicts of interest regarding the publication of this paper.

\section{Authors' Contributions}

The authors contributed equally to this paper. All authors read and approved the final manuscript.

\section{Acknowledgments}

This work was supported by the NSFC (no. 11361054, no. 11671322).

\section{References}

[1] C. P. Gupta, "Existence and uniqueness theorems for the bending of an elastic beam equation," Applicable Analysis. An International Journal, vol. 26, no. 4, pp. 289-304, 1988.

[2] R. A. Usmani, "A uniqueness theorem for a boundary value problem," Proceedings of the American Mathematical Society, vol. 77, no. 3, pp. 329-335, 1979.

[3] J. Schröder, "Operator inequalities," in Mathematics in Science and Engineering, vol. 147, Academic Press, Inc., Cambridge, Mass, USA, 1980.

[4] Y. S. Yang, "Fourth-order two-point boundary value problems," Proceedings of the American Mathematical Society, vol. 104, no. 1, pp. 175-180, 1988.

[5] R. P. Agarwal, "On fourth order boundary value problems arising in beam analysis," Differential and Integral Equations. An International Journal for Theory and Applications, vol. 2, no. 1, pp. 91-110, 1989.

[6] M. A. del Pino and R. F. Manasevich, "Existence for a fourthorder boundary value problem under a two-parameter nonresonance condition," Proceedings of the American Mathematical Society, vol. 112, no. 1, pp. 81-86, 1991.

[7] R. Ma and H. Wang, "On the existence of positive solutions of fourth-order ordinary differential equations," Applicable Analysis, vol. 59, no. 1-4, pp. 225-231, 1995.

[8] Z. Bai and H. Wang, "On positive solutions of some nonlinear fourth-order beam equations," Journal of Mathematical Analysis and Applications, vol. 270, no. 2, pp. 357-368, 2002.

[9] Y. Li, "Positive solutions of fourth-order boundary value problems with two parameters," Journal of Mathematical Analysis and Applications, vol. 281, no. 2, pp. 477-484, 2003. 
[10] P. Drábek, G. Holubová, A. s. Matas, and P. Necesal, "Nonlinear models of suspension bridges: discussion of the results," Applications of Mathematics, vol. 48, no. 6, pp. 497-514, 2003.

[11] B. Liu, "Positive solutions of fourth-order two point boundary value problems," Applied Mathematics and Computation, vol. 148, no. 2, pp. 407-420, 2004.

[12] C. De Coster and P. Habets, "The lower and upper solutions method for boundary value problems," in Handbook of Differential Equations: Ordinary Differential Equations, A. Cañada, P. Drábek, and A. Fonda, Eds., vol. 1, pp. 69-160, 2004.

[13] Q. Yao, "Existence, multiplicity and infinite solvability of positive solutions to a nonlinear fourth-order periodic boundary value problem," Nonlinear Analysis. Theory, Methods \& Applications, vol. 63, no. 2, pp. 237-246, 2005.

[14] G. Han and F. Li, "Multiple solutions of some fourth-order boundary value problems," Nonlinear Analysis. Theory, Methods \& Applications, vol. 66, no. 11, pp. 2591-2603, 2007.

[15] A. Cabada, J. Á. Cid, and L. s. Sanchez, "Positivity and lower and upper solutions for fourth order boundary value problems," Nonlinear Analysis. Theory, Methods \& Applications. An International Multidisciplinary Journal, vol. 67, no. 5, pp. 1599-1612, 2007.

[16] G. Han and Z. Xu, "Multiple solutions of some nonlinear fourthorder beam equations," Nonlinear Analysis. Theory, Methods \& Applications, vol. 68, no. 12, pp. 3646-3656, 2008.

[17] J. R. Webb, G. Infante, and D. Franco, "Positive solutions of nonlinear fourth-order boundary-value problems with local and non-local boundary conditions," Proceedings of the Royal Society of Edinburgh. Section A. Mathematics, vol. 138, no. 2, pp. 427-446, 2008.

[18] B. P. Rynne, "Infinitely many solutions of superlinear fourth order boundary value problems," Topological Methods in Nonlinear Analysis, vol. 19, no. 2, pp. 303-312, 2002.

[19] R. Ma, C. Gao, and X. Han, "On linear and nonlinear fourthorder eigenvalue problems with indefinite weight," Nonlinear Analysis. Theory, Methods \& Applications, vol. 74, no. 18, pp. 6965-6969, 2011.

[20] G. Dai and X. Han, "Global bifurcation and nodal solutions for fourth-order problems with sign-changing weight," Applied Mathematics and Computation, vol. 219, no. 17, pp. 9399-9407, 2013.

[21] J. Wang and R. Ma, "S-shaped connected component for the fourth-order boundary value problem," Boundary Value Problems, vol. 2016, 189 pages, 2016.

[22] I. Sim and S. Tanaka, "Three positive solutions for onedimensional $p$-Laplacian problem with sign-changing weight," Applied Mathematics Letters, vol. 49, pp. 42-50, 2015.

[23] G. T. Whyburn, Topological Analysis, Princeton University Press, Princeton, NJ, USA, 1958.

[24] R. Ma and Y. An, "Global structure of positive solutions for superlinear second order $m$-point boundary value problems," Topological Methods in Nonlinear Analysis, vol. 34, no. 2, pp. 279-290, 2009. 


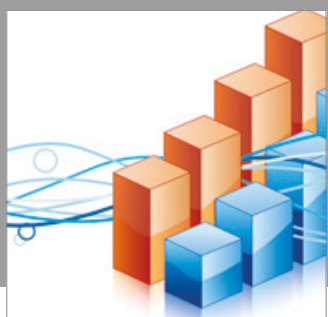

Advances in

Operations Research

vatersals

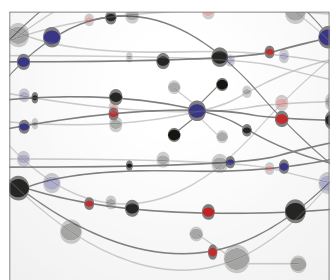

\section{The Scientific} World Journal
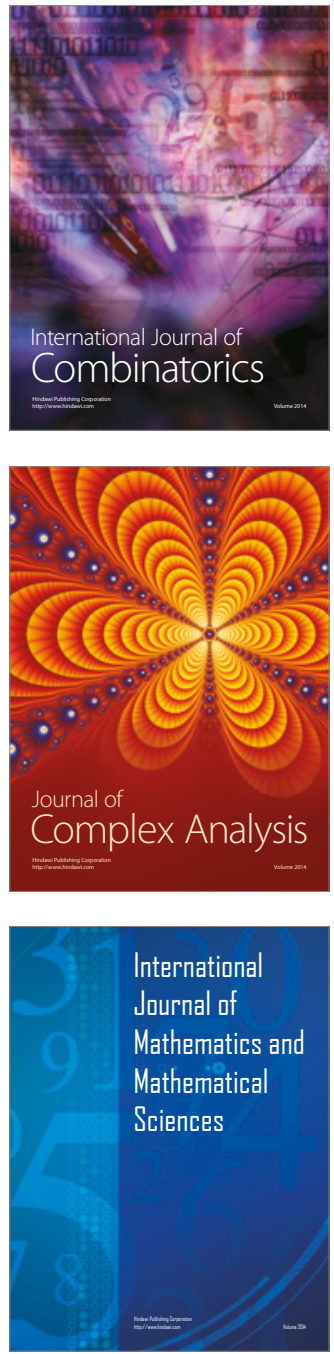
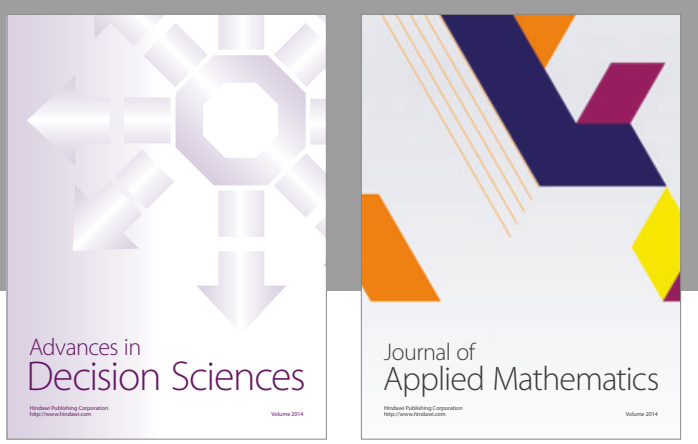

Algebra

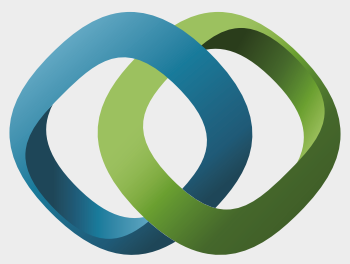

\section{Hindawi}

Submit your manuscripts at

https://www.hindawi.com
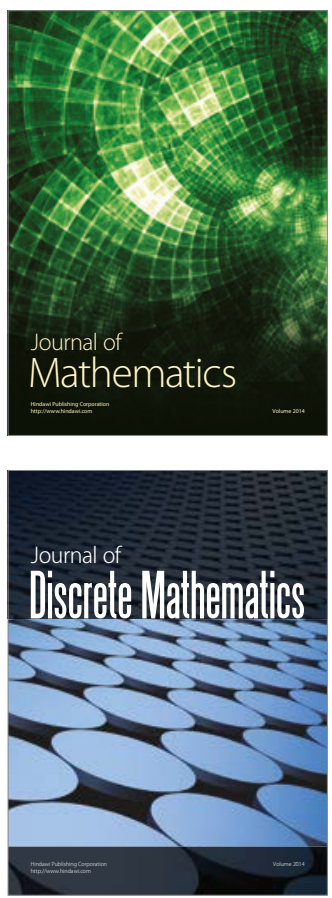

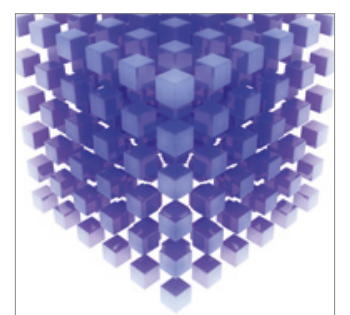

Mathematical Problems in Engineering
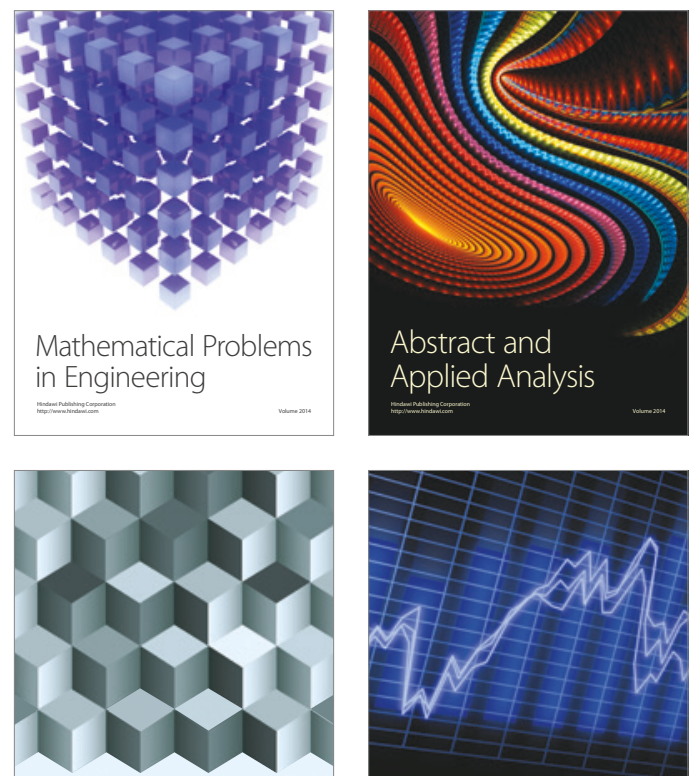

Journal of

Function Spaces

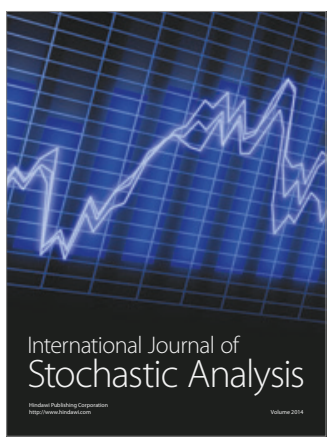

Probability and Statistics
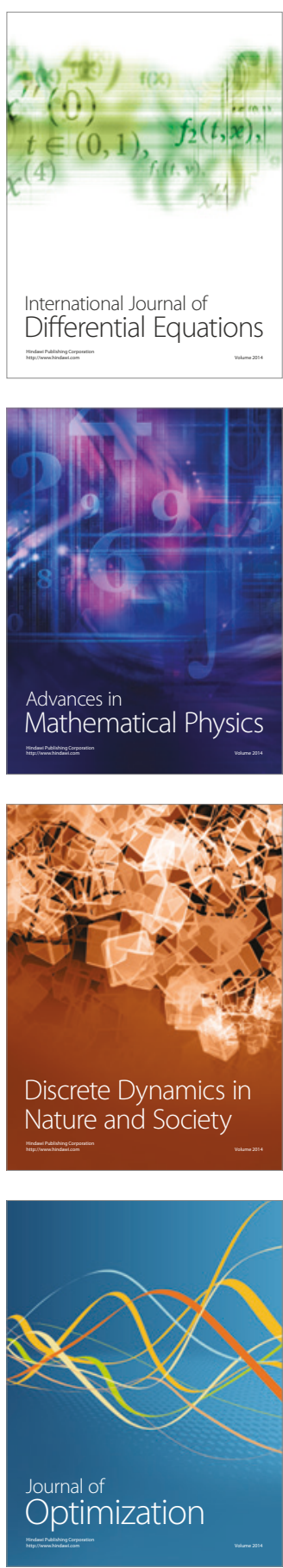